\title{
A considerable study about the DNA dynamics arising in oscillator-chain of Peyrard-Bishop model
}

\author{
khalid k. Ali ${ }^{1}$ \\ 1 al-azhar unifersty \\ September 16, 2020
}

\begin{abstract}
In this work, the study of the Peyrard-Bishop DNA dynamic model equation analytically and numerically will present. The Kudryashov method and modified Kudryashov method are used to nd the solution of the Peyrard-Bishop DNA dynamic model equation analytically. A cubic B-spline collocation method is used to obtain a numerical solution of the Peyrard-Bishop DNA dynamic model equation. A comparison between the results obtained by the analytical methods and the numerical method is investigated. We give some figures to show how accurate the solutions will be obtained from analytical and numerical methods.
\end{abstract}

\section{Hosted file}

DNA 2020..pdf available at https://authorea.com/users/359651/articles/481488-a-considerablestudy-about-the-dna-dynamics-arising-in-oscillator-chain-of-peyrard-bishop-model 